\title{
Post meningitis profound hearing loss: MRI as a predictor tool for urgent cochlear implant
}

\author{
Miura MS*, Mendonça AJC, Soccol RA and MN Rios \\ Department of Medical Surgery of Universidade Federal de Ciências da Saúde de Porto Alegre and Division of Pediatric Otorhinolaryngology at Hospital da Criança \\ Santo Antônio, Programa de Pós-Graduação em Ciências Médicas-Faculdade de Medicina da Universidade Federal do Rio Grande do Sul, Porto Alegre, Brazil
}

\begin{abstract}
Bacterial meningitis is the most common cause of acquired sensorineural hearing loss in children, once it could quickly trigger ossification of cochlea, known as labyrinthitis ossificans. Medical team should be aware of such potential threaten and monitor hearing function of these patients. On suspicion of hearing loss, it is urgent to proceed audiological and radiological evaluation before complete cochlear ossification, since it evolves to profound hearing loss and reduces successful cochlear implant insertion. Although CT scan detect cochlear ossification, it sometimes too late. More recently, MRI could detect early intracochlear labyrinthitis ossificans stages, allowing cochlear implant surgery in the right moment.
\end{abstract}

\section{Introduction}

Bacterial meningitis (BM) is the most common cause of acquired sensorineural hearing loss (SNHL) in children [1,2-9,10-12]. 5-7\% of patients will develop hearing loss, since meningitis induces to labyrinthitis ossificans (LO) [7]. This process can be quick, and the medical team should monitor hearing function of these patients, as well as guide parents / caregivers for any disorders related to speech, language, responses to environmental sounds, or behavior changes. On suspicion of hearing loss, it is urgent to proceed audiological and radiological evaluation.

\section{Case report}

1 year and 8 months old boy was referred to our cochlear implant program six weeks after discharge due to Streptococcus pneumoniae meningitis. During hospitalization, associated to meningitis, he presented left side transient peripheral facial paralysis and gait abnormalities, falling to the left side. 2 weeks after discharge, parents observed hearing impairment with words and sounds; he stopped talking and couldn't respond to verbal commands anymore, as well as he did not follow animated movies and cartoons. An audiological evaluation was performed, with absent otoacoustic emissions and ABR without presence of waves. After two weeks, he was referred to our Hospital and quickly performed ABR tone-burst and radiological evaluation. Tone burst confirmed left ear's absence of waves and presence of only wave $\mathrm{V}(100 \mathrm{dBNA})$ in $500 \mathrm{~Hz}$ and $2 \mathrm{kHz}$ in the right ear. In CT scan of temporal bone there was no cochlear disease, however ossification of lateral semicircular canals was identified (Figure 1). MRI of temporal bone demonstrated in T2 and Fiesta an irregular impregnation of left cochlea and signal loss in the lateral semicircular canals (Figure 2). He underwent surgery for cochlear implant after 2 weeks and intraoperative findings confirmed ossification in scala tympani; right side's disobliteration was achieved with full insertion of electrode array (Figure 3). In left side, presence of advanced ossification allowed partial disobliteration and insertion of 14 electrodes (Figure 4).

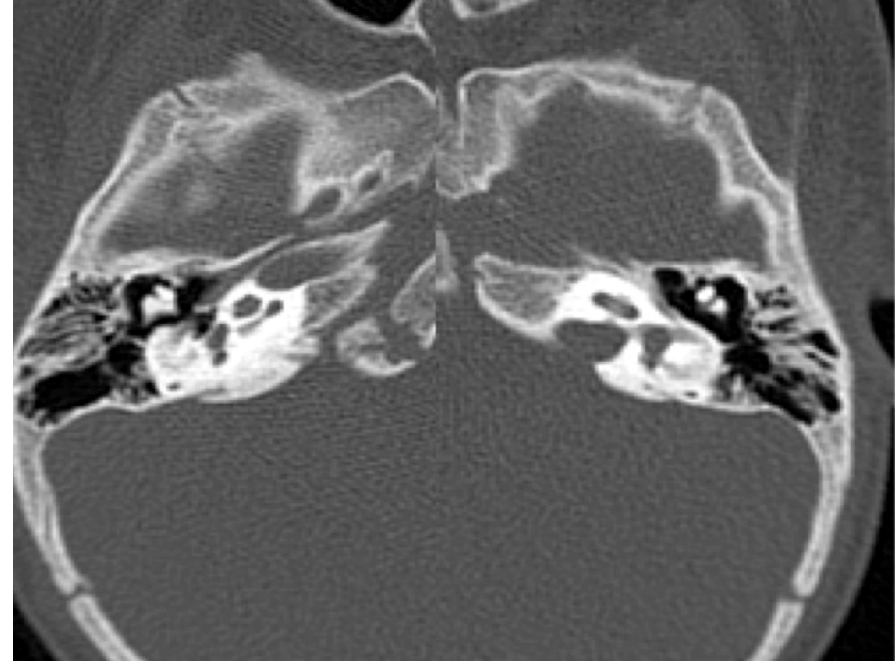

Figure 1. CT scan: ossification of lateral semicircular canals

\section{Discussion}

$\mathrm{BM}$ is the main triggering of $\mathrm{LO}(79 \%)$, manifesting as acquired SNHL [1]. LO of bacterial etiology may spread by three routes: meningeal, tympanic or hematogenic [1,3]. Acute inflammatory response into membranous labyrinth often leads to fibrosis and finally the ossification [1]. Isolated involvement of scala tympani in cochlear basal turn is a common finding $[1,3]$. In general, ossification

${ }^{\star}$ Correspondence to: Miura MS, Department of Medical Surgery of Universidade Federal de Ciências da Saúde de Porto Alegre and Division of Pediatric Otorhinolaryngology at Hospital da Criança Santo Antônio, Programa de PósGraduação em Ciências Médicas-Faculdade de Medicina da Universidade Federal do Rio Grande do Sul, Porto Alegre, Brazil, E-mail: mmiura.voy@terra.com.br

Received: June 29, 2018 Accepted: July 09, 2018; Published: July 12, 2018 


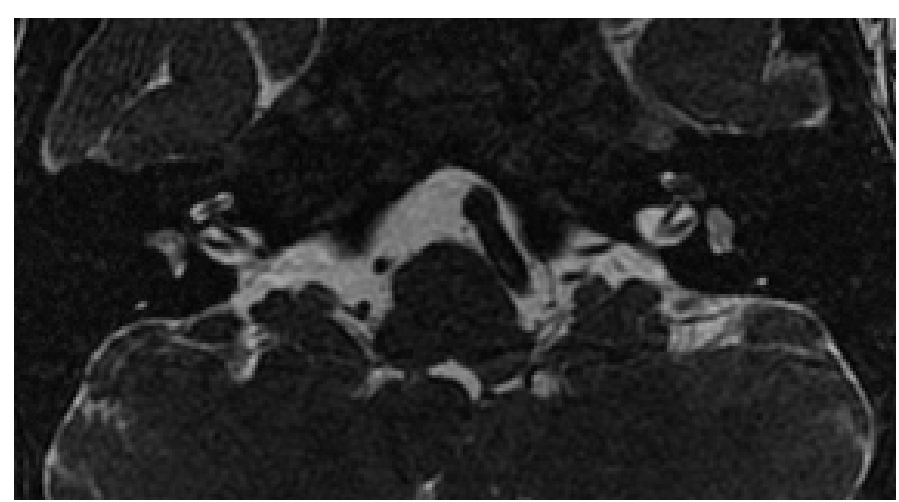

Figure 2. MRI: irregular impregnation of left cochlea and signal loss in the lateral semicircular canals

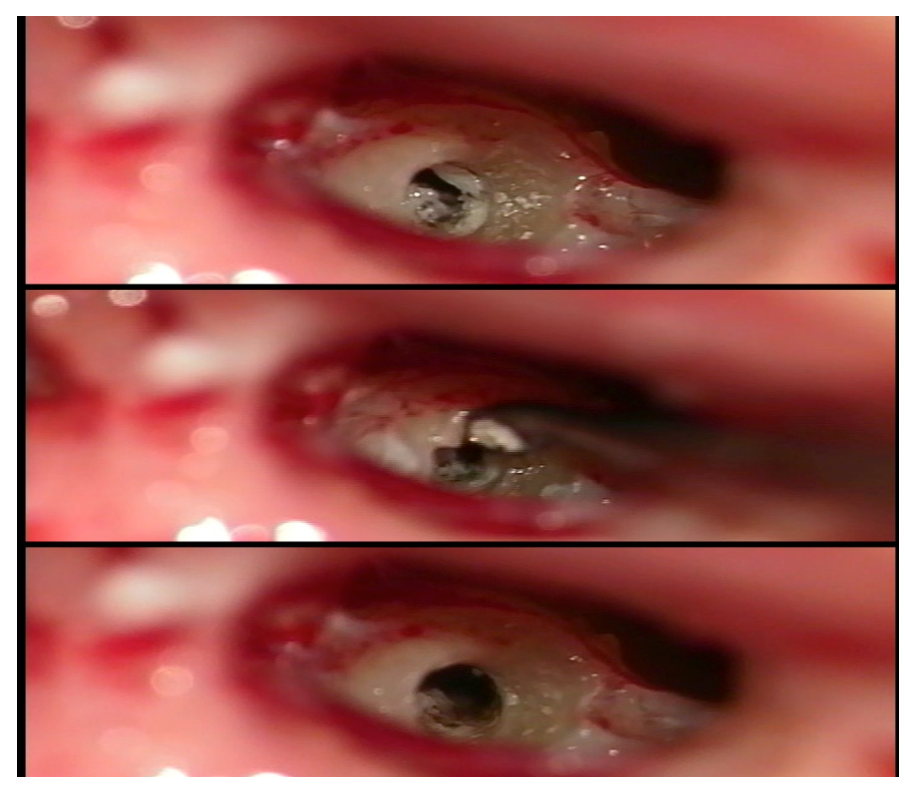

Figure 3. Right cochleostomy from top to bottom: intraoperative finding of scala tympani ossification; curette disobliteration; and complete opening of scala tympani

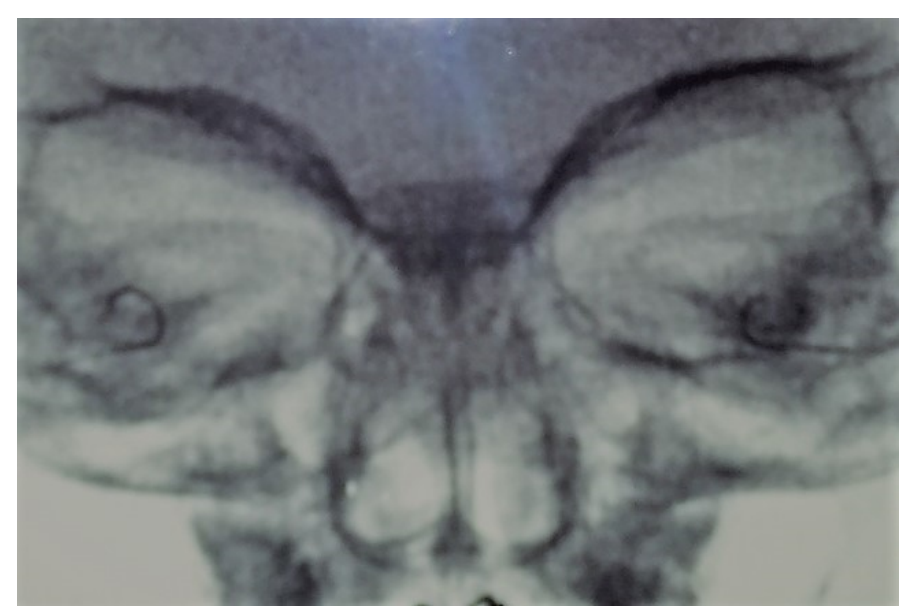

Figure 4. Post-operative X-Ray: right cochlear full electrode array insertion; left cochlear partial electrode array insertion

development begins in peri-lymphatic space of cochlear basal turn and may eventually involve entire inner ear [3]. In our report, first symptoms were vestibular, and imaging confirmed ossification of lateral semicircular canals, with subsequent cochlear involvement.
Recognized risk factors for developing SNHL are: S. pneumoniae infection, prolonged hospitalization, seizures, CSF analysis with high protein or low glucose [1]. SNHL have been documented as soon as 48 hours after admission for BM [1] and ossification process can occur as early as 2 weeks following onset of infection [7]. In this child, parents noticed behavioral change, interruption of language development, speech regression and lack of response to loud sounds, looking for medical evaluation quickly. ABR tone-burst confirmed hearing loss, unresponsive in left ear and residual response in right ear.

High resolution CT scan has been the method of choice to demonstrate LO by ossification visualization within the otic capsule $[1,7]$.

More recently, T2 MRI sequences has shown high sensitivity in the diagnosis of LO presumably by identifying intra-labyrinthine fibrosis that occurs prior to the ossification. MRI has high sensitivity (92.8\%) and high positive predictive value (81.2\%) to identify intra-cochlear LO [1]. Booth et al. demonstrated high correlation $(r=0.721)$ between the degree of ossification and intraoperative MRI findings. Labyrinthine highlight can be seen on T1 image contrasted with gadolinium in acute LO. Signal loss on T2 sequences are related to fibrous bone changes in the inner ear [2]. In CT scan, only delayed bone changes associated with LO can be seen, and scar extension may be underestimated [2]. In our Hospital, it is possible simultaneous CT scan and MRI examination in young children under general anesthesia. Despite the audiological indications of cochlear involvement, CT scan was not yet compatible, which could have delayed our decision of urgent realization of simultaneous bilateral cochlear implant. Fortunately, MRI confirmed early cochlear involvement by LO, most advanced on left side, correlated with surgical findings.

Typically, scala tympani is the ideal location for insertion of electrodes of cochlear implant, due to high concentration of sensory nerve endings. It should be noted that surgical team must be prepared to deal with obliteration of the scala tympani; it is possible to disobliterate by curettage and drilling, as well as use of electrode with stylet, or if unavailable, using a rigid curved probe to ensure further advancement of electrodes. However, if there is no lumen patency, vestibular scale could be a viable location [4]. Extensive involvement of the scala tympani in the basal and upward turn of the cochlea with possible involvement of the middle turn may require alternative insertion technique of electrodes, as an insert in the scala vestibuli, double array electrode or circummodiolar drilling [7]. Our option was cochleostomy and, despite ossification, removal with curette was possible, achieving full insertion of electrodes in right cochlea and partial insertion in left cochlea (14 electrodes). The child had excellent post-implantation recovery, with activation in three weeks, and hearing deprivation time of 60 days.

\section{Conclusion}

Evolution to SNHL after meningitis may occur early and suggests the development of LO. S. pneumoniaeis the most likely causative agent in the presence of profound hearing loss. MRI is the method of choice for early diagnosis, once CT scan has low sensitivity (40-50\%) in detection of initial stages of ossification. Ossification signs in patient with severe to profound hearing loss represents an urgency to cochlear implant, because in some week's ossification can prevent the insertion of electrodes, definitely compromising the future of these patient hearing. It is important surgical technique planning to address the ossification, as well as the proper image investigation through MRI. 


\section{References}

1. Booth TN, Roland P, Kutz JW Jr, Lee K, Isaacson B (2013) High-resolution 3-D T2-weighted imaging in the diagnosis of labyrinthitis ossificans: emphasis on subtle cochlear involvement. Pediatr Radiol 43: 1584-1590. [Crossref]

2. Kaya S, Paparella MM, Cureoglu S (2016) Pathologic Findings of the Cochlea in Labyrinthitis Ossificans Associated with the Round Window Membrane. Otolaryngol Head Neck Surg 155: 635-640. [Crossref]

3. Dhanjal H, Rainsbury J, Irving RM (2014) Bilateral sensorineural hearing loss and labyrinthitis ossificans secondary to neurosarcoidosis. Cochlear Implants Int 15: 337 340. [Crossref]

4. Liu CC, Sweeney M, Booth TN, Lee KH, Kutz JW, et al. (2015) The Impact of Postmeningitic Labyrinthitis Ossificans on Speech Performance After Pediatric Cochlear Implantation. Otol Neurotol 36: 1633-1637. [Crossref]

5. Douglas SA, Sanli H, Gibson WP (2008) Meningitis resulting in hearing loss and labyrinthitis ossificans - does the causative organism matter? Cochlear Implants Int 9: 90-96. [Crossref]
6. Isaacson B, Booth T, Kutz JW Jr, Lee KH, Roland PS (2009) Labyrinthitis ossificans: how accurate is MRI in predicting cochlear obstruction? Otolaryngol Head Neck Surg 140: 692-696. [Crossref]

7. Jiang ZY, Odiase E, Isaacson B, Roland PS, Kutz JW Jr (2014) Utility of MRIs in adult cochlear implant evaluations. Otol Neurotol 35: 1533-1535. [Crossref]

8. Chan CC, Saunders DE, Chong WK, Hartley BE, Raglan E, et al. (2007) Advancemen in post-meningitic lateral semicircular canal labyrinthitis ossificans. J Laryngol Otol 121: 105-109. [Crossref]

9. Ashfield T, Pai I, Wilson K, Britz A, Connor S, et al. (2015) Cochlear implantation in children with sickle cell disease. Pediatr Int 57: 174-176. [Crossref]

10. Wasson JD, Briggs RJ (2016) Contemporary surgical issues in paediatric cochlear implantation. Int J Audiol 55 Supp1 2: S77-87. [Crossref]

11. Angeli SI, Goncalves S (2016) Predicting depth of electrode insertion by cochlear measurements on computed tomography scans. Laryngoscope 126: 1656-1661. [Crossref]

12. Lambert PR, Ruth RA, Hodges AV (1991) Multichannel cochlear implant and electrically evoked auditory brainstem responses in a child with labyrinthitis ossificans. Laryngoscope 101: 14-19.

Copyright: (C2018 Miura MS. This is an open-access article distributed under the terms of the Creative Commons Attribution License, which permits unrestricted use, distribution, and reproduction in any medium, provided the original author and source are credited. 